\title{
Existence of positive stationary solutions for a reaction-diffusion system
}

Fanglei Wang $^{1 *}$ and Yunhai Wang ${ }^{2}$

${ }^{*}$ Correspondence:
wang-fanglei@hotmail.com
${ }^{1}$ College of Science, Hohai
University, Nanjing, 210098, P.R.
China
Full list of author information is
available at the end of the article

\section{Abstract}

In this paper, we will establish some existence results of positive stationary solutions for a reaction-diffusion system

$$
\left\{\begin{array}{l}
-u^{\prime \prime}+\lambda u=p(t) \varphi u+h(t) f(u, \varphi), \quad 0<t<1 \\
-\varphi^{\prime \prime}=q(t) u^{\alpha}, \quad 0<t<1 \\
u(0)=u(1)=0 \\
\varphi(0)=\varphi(1)=0
\end{array}\right.
$$

The main method used here is the well-known fixed point theorem of cone expansion and compression.

MSC: $34 \mathrm{~B} 10$

Keywords: reaction-diffusion system; stationary solutions; fixed point theorem

\section{Introduction}

The purpose of this work is to study the existence of positive solutions for the following system:

$$
\left\{\begin{array}{l}
-u^{\prime \prime}+\lambda u=p(t) \varphi u+h(t) f(u, \varphi), \quad 0<t<1, \\
-\varphi^{\prime \prime}=q(t) u^{\alpha}, \quad 0<t<1, \\
u(0)=u(1)=0 \\
\varphi(0)=\varphi(1)=0
\end{array}\right.
$$

where $\lambda>-\pi^{2}, \alpha>0$ are constants. By a positive solution, we mean a pair of functions $(u, \varphi)$ with $u, \varphi \in C^{2}(0,1) \cap C[0,1]$ is a positive solution of (1), if $(u, \varphi)$ satisfies (1), and $u, \varphi \geq 0, t \in[0,1], u, \varphi \not \equiv 0$.

During the last few decades, concerning local equations, the question of the existence of solutions is one of the important topics, and it therefore has attracted much attention [1-7]. Usually, the proof is based on either topological methods or a variational approach. For example, in [1], Gu and Wang applied an abstract fixed point theorem and the estimate of the weighted $L^{p}$ norm to study a reaction-diffusion system as

(c) 2016 Wang and Wang. This article is distributed under the terms of the Creative Commons Attribution 4.0 International License (http://creativecommons.org/licenses/by/4.0/), which permits unrestricted use, distribution, and reproduction in any medium, provided you give appropriate credit to the original author(s) and the source, provide a link to the Creative Commons license, and indicate if changes were made. 
follows:

$$
\left\{\begin{array}{l}
u_{1 t}-\Delta u_{1}=u_{1} u_{2}-b u_{1}, \quad x \in \Omega, t>0, \\
u_{2 t}-\Delta u_{2}=a u_{1}, \quad x \in \Omega, t>0, \\
u_{1}=u_{2}=0, \quad x \in \partial \Omega, t>0, \\
u_{1}(x, 0)=u_{10}(x) \geq 0, \quad u_{2}(x, 0)=u_{20}(x) \geq 0, \quad x \in \bar{\Omega},
\end{array}\right.
$$

where $\Omega \in R^{N}$ is a smooth bounded domain, $a, b>0$ are constants and $u_{10}, u_{20}$ are continuous nonnegative functions on $\bar{\Omega}$. In [2], by using the fixed point theorem of cone expansion and compression, Wang and An investigated the existence and multiplicity of positive solutions for the following system:

$$
\left\{\begin{array}{l}
-u^{\prime \prime}+\lambda u=\varphi u+h(t) f(u), \quad 0<t<1, \\
-\varphi^{\prime \prime}=\mu u, \quad 0<t<1, \\
u(0)=u(1)=0, \quad \varphi(0)=\varphi(1)=0 .
\end{array}\right.
$$

More recently, Chen and Ma obtained some existence results for (1) using the bifurcation techniques in [3, 4]. In addition, in [5-7], the authors used the variational approach to study the existence, nonexistence, multiplicity, and qualitative behavior of the solutions in the semiclassical limit for the Schrödinger-Poisson system as follows:

$$
\left\{\begin{array}{l}
-\Delta u+V(x) u=K_{1}(x) \phi u+u^{p} \\
-\Delta \phi=K_{2}(x) u^{2}
\end{array}\right.
$$

where $1<p<5$.

We are mainly motivated by the recent nice works $[2,8,9]$ and focus on the general local equations (1): (a) the first equation of (1) has an external force such as $f(u, \varphi)$; (b) the second equation of (1) has the nonlinearity of arbitrary growth $u^{\alpha}$. By employing the fixed point theorem of cone expansion and compression, we will establish some existence results of positive solutions for (1), if the exponent $\alpha$ satisfies two cases: (i) $\alpha \geq 1$; (ii) $0<\alpha<1$.

The remaining part of this paper is organized as follows. In Section 2 , some preliminary results are given. In Section 3, we show the main results. In Section 4, to illustrate the new results, some applications are also given.

\section{Preliminaries}

Let us describe some known results established by Li in [11].

Lemma 2.1 If $\lambda>-\pi^{2}$, then the linear boundary value problem

$$
-u^{\prime \prime}+\lambda u=0, \quad u(0)=u(1)=0,
$$

has a nonnegative Green function $G(t, s)$.

Lemma 2.2 The function $G(t, s)$ has the following properties:

(i) $G(t, s)>0, \forall t, s \in(0,1)$; 
(ii) $G(t, s) \leq C G(s, s), \forall t, s \in[0,1]$;

(iii) $G(t, s) \geq \delta G(t, t) G(s, s), \forall t, s \in[0,1]$.

For convenience, we assume the following conditions hold throughout this paper:

(H1) $q(t), p(t):[0,1] \rightarrow[0,+\infty)$ are continuous, and satisfy

$$
C \int_{0}^{1} \int_{0}^{1} G(s, s) K(s, \tau) p(s) q(\tau) d \tau d s<\frac{1}{2},
$$

where $K(t, s)$ denotes the Green function $G(t, s)$ when $\lambda=0$;

$(\mathrm{H} 2) h(t):[0,1] \rightarrow[0,+\infty)$ is continuous, and

$$
0<\int_{0}^{1} G(s, s) h(s) d s<\infty
$$

Using the idea in [2], Lemma 2.1, and Lemma 2.2, it is easy to convert system (1) into a fixed point equation as follows:

$$
\begin{aligned}
u(t)= & \int_{0}^{1} \int_{0}^{1} G(t, s) K(s, \tau) p(s) q(\tau) u(s) u^{\alpha}(\tau) d \tau d s \\
& +\int_{0}^{1} G(t, s) h(s) f\left(u(s), \int_{0}^{1} K(s, \tau) q(\tau) u^{\alpha}(\tau) d \tau\right) d s
\end{aligned}
$$

Now define a mapping $T: C[0,1] \rightarrow C[0,1]$ by

$$
\begin{aligned}
T u(t)= & \int_{0}^{1} \int_{0}^{1} G(t, s) K(s, \tau) p(s) q(\tau) u(s) u^{\alpha}(\tau) d \tau d s \\
& +\int_{0}^{1} G(t, s) h(s) f\left(u(s), \int_{0}^{1} K(s, \tau) q(\tau) u^{\alpha}(\tau) d \tau\right) d s,
\end{aligned}
$$

and define a positive cone $P \subset C[0,1]$ as

$$
P=\left\{u(t) \in C[0,1]: \min _{t \in[\theta, 1-\theta]} u(t) \geq \sigma\|u\|\right\}
$$

where $\theta \in\left(0, \frac{1}{2}\right)$ is a fixed constant, $\sigma \in(0,1)$.

Through a standard proof process, we have the following.

Lemma 2.3 Assume that (H1)-(H2) hold. Then $T(P) \subset P$ and $T: P \rightarrow P$ is completely continuous.

The proofs of our main results are based on the following fixed point, which can be found in [10].

Lemma 2.4 Let $E$ be a Banach space, and $K \subset E$ be a cone in $E$. Assume $\Omega_{1}, \Omega_{2}$ are open subsets of $E$ with $0 \in \Omega_{1}, \bar{\Omega}_{1} \subset \Omega_{2}$, and let $T: K \cap\left(\bar{\Omega}_{2} \backslash \Omega_{1}\right) \rightarrow K$ be a completely continuous operator such that either:

(i) $\|T u\| \leq\|u\|, u \in K \cap \partial \Omega_{1}$ and $\|T u\| \geq\|u\|, u \in K \cap \partial \Omega_{2}$; or

(ii) $\|T u\| \geq\|u\|, u \in K \cap \partial \Omega_{1}$ and $\|T u\| \leq\|u\|, u \in K \cap \partial \Omega_{2}$.

Then $T$ has a fixed point in $K \cap\left(\bar{\Omega}_{2} \backslash \Omega_{1}\right)$. 


\section{Main result}

Theorem 3.1 Assume (H1)-(H2) hold, and $\alpha \geq 1$. In addition, the following conditions hold:

(H3) $q(t)$ satisfies

$$
\min _{t \in[\theta, 1-\theta]} \int_{\theta}^{1-\theta} K(t, s) q(s) d s>0
$$

(H4) $h(t)$ satisfies

$$
0<\int_{\theta}^{1-\theta} G\left(\frac{1}{2}, s\right) h(s) d s
$$

(H5) $f \in C\left[R^{+} \times R^{+}, R^{+}\right]$, and $f(u, \varphi)$ are nondecreasing in $u$ and $\varphi$ for $u, \varphi>0$, moreover,

$$
\left\{\begin{array}{l}
0<u \leq \bar{u}, \\
0<\varphi \leq \bar{\varphi},
\end{array} \quad \Rightarrow \quad f(u, \varphi) \leq f(\bar{u}, \bar{\varphi}),\right.
$$

in addition, $f(u, \varphi)$ satisfies the following conditions:

$$
\lim _{s \rightarrow \infty} \frac{f\left(s, m_{1} s\right)}{s}=\infty, \quad \lim _{s \rightarrow 0} \frac{f\left(s, M_{1} s\right)}{s}=0,
$$

where

$$
m_{1}=\sigma^{\alpha-1} \min _{s \in[\theta, 1-\theta]} \int_{\theta}^{1-\theta} K(s, \tau) q(\tau) d \tau, \quad M_{1}=\int_{0}^{1} K(\tau, \tau) q(\tau) d \tau .
$$

Then system (1) has at least one positive solution.

Proof On one hand, since $\lim _{s \rightarrow 0} \frac{f\left(s, M_{1} s\right)}{s}=0$, there exists a $\eta>0$ with $\eta<1$ such that

$$
f\left(s, M_{1} s\right) \leq \epsilon s, \quad \text { for } 0<s<\eta
$$

where $\epsilon$ satisfies

$$
\epsilon C \int_{0}^{1} G(s, s) h(s) d s \leq \frac{1}{2} .
$$

Taking $r \in(0, \eta)$ and setting $\Omega_{r}=\{u(t) \in C[0,1]:\|u\|<r\}$, then, for any $u(t) \in \partial \Omega_{r} \cap P$, we have

$$
\begin{aligned}
T u(t)= & \int_{0}^{1} \int_{0}^{1} G(t, s) K(s, \tau) p(s) q(\tau) u(s) u^{\alpha}(\tau) d \tau d s \\
& +\int_{0}^{1} G(t, s) h(s) f\left(u, \int_{0}^{1} K(s, \tau) q(\tau) u^{\alpha}(\tau) d \tau\right) d s \\
= & C \int_{0}^{1} \int_{0}^{1} G(s, s) K(s, \tau) p(s) q(\tau) d \tau d s \cdot r^{1+\alpha} \\
& +C \int_{0}^{1} G(s, s) h(s) f\left(r, \int_{0}^{1} K(\tau, \tau) q(\tau) d \tau \cdot r^{\alpha}\right) d s
\end{aligned}
$$




$$
\begin{aligned}
& <\frac{1}{2} r^{1+\alpha}+C \int_{0}^{1} G(s, s) h(s) f\left(r, M_{1} r^{\alpha}\right) d s \\
& \leq \frac{1}{2} r+C \int_{0}^{1} G(s, s) h(s) f\left(r, M_{1} r\right) d s \\
& \leq \frac{1}{2} r+\epsilon r C \int_{0}^{1} G(s, s) h(s) d s \\
& \leq r=\|u\| .
\end{aligned}
$$

Consequently,

$\|T u\|<\|u\|, \quad \forall u \in \partial \Omega_{r} \cap P$.

On the other hand, since $\lim _{s \rightarrow \infty} \frac{f\left(s, m_{1} s\right)}{s}=\infty$, there exists a $\bar{R}>0$ such that

$$
f\left(s, m_{1} s\right) \geq \rho s, \quad \forall s \geq \bar{R}
$$

where $\rho$ satisfies

$$
\rho \sigma \int_{\theta}^{1-\theta} G\left(\frac{1}{2}, s\right) h(s) d s>1 .
$$

Choosing $R>\left\{\frac{\bar{R}}{\sigma}, 2\right\}$, and setting $\Omega_{R}=\{u \in C[0,1]:\|u\|<R\}$, it is easy to verify that

$$
\min _{t \in[\theta, 1-\theta]} u(t) \geq \sigma\|u\|=\sigma R>\bar{R}, \quad \forall u(t) \in \partial \Omega_{R} \cap P
$$

Then, for any $u(t) \in \partial \Omega_{R} \cap P$, we have

$$
\begin{aligned}
T u\left(\frac{1}{2}\right)= & \int_{0}^{1} \int_{0}^{1} G\left(\frac{1}{2}, s\right) K(s, \tau) p(s) q(\tau) u(s) u^{\alpha}(\tau) d \tau d s \\
& +\int_{0}^{1} G\left(\frac{1}{2}, s\right) h(s) f\left(u, \int_{0}^{1} K(s, \tau) q(\tau) u^{\alpha}(\tau) d \tau\right) d s \\
\geq & \int_{0}^{1} G\left(\frac{1}{2}, s\right) h(s) f\left(u, \int_{0}^{1} K(s, \tau) q(\tau) u^{\alpha}(\tau) d \tau\right) d s \\
\geq & \int_{\theta}^{1-\theta} G\left(\frac{1}{2}, s\right) h(s) f\left(u, \int_{0}^{1} K(s, \tau) q(\tau) u^{\alpha}(\tau) d \tau\right) d s \\
\geq & \int_{\theta}^{1-\theta} G\left(\frac{1}{2}, s\right) h(s) f\left(u, \int_{\theta}^{1-\theta} K(s, \tau) q(\tau) u^{\alpha}(\tau) d \tau\right) d s \\
\geq & \int_{\theta}^{1-\theta} G\left(\frac{1}{2}, s\right) h(s) f\left(\sigma\|u\|, \sigma^{\alpha}\|u\|^{\alpha} \min _{s \in[\theta, 1-\theta]} \int_{\theta}^{1-\theta} K(s, \tau) q(\tau) d \tau\right) d s \\
\geq & \int_{\theta}^{1-\theta} G\left(\frac{1}{2}, s\right) h(s) f\left(\sigma\|u\|, \sigma^{\alpha-1} \min _{s \in[\theta, 1-\theta]} \int_{\theta}^{1-\theta} K(s, \tau) q(\tau) d \tau \cdot \sigma\|u\|\right) d s \\
= & \int_{\theta}^{1-\theta} G\left(\frac{1}{2}, s\right) h(s) f\left(\sigma\|u\|, m_{1} \sigma\|u\|\right) d s \\
\geq & \int_{\theta}^{1-\theta} G\left(\frac{1}{2}, s\right) h(s) \rho \sigma\|u\| d s>\|u\| . \\
&
\end{aligned}
$$


Consequently,

$$
\|T u\|>\|u\|, \quad \forall u(t) \in \partial \Omega_{R} \cap P .
$$

Then by Lemma 2.4, system (1) has a positive solution $\left(u(t), \int_{0}^{1} K(t, s) q(s) u^{\alpha}(s) d s\right)$.

In the condition (H5) of Theorem 3.1, the function $f(u, \varphi)$ is required to be nondecreasing in $u$ and $\varphi$ for $u, \varphi>0$. If the function $f(u, \varphi)$ is mixed monotone in the condition $(\mathrm{H} 7)$, we impose a stronger requirement on the function $h(t)$ in the condition (H6). Then, similar to the proof of Theorem 3.1, we can obtain the following theorem.

Theorem 3.2 Assume (H1)-(H4) hold, and $\alpha \geq 1$. In addition, the following conditions hold:

(H6) $h(t)$ satisfies $h(t) \equiv 0$ for $t \in[0, \theta] \cup[1-\theta, 1]$, and

$$
0<\int_{\theta}^{1-\theta} G\left(\frac{1}{2}, s\right) h(s) d s
$$

(H7) $f \in C\left[R^{+} \times R^{+}, R^{+}\right]$, and $f(u, \varphi)$ is nonincreasing in $u$ and nondecreasing in $\varphi$ for $u, \varphi>0$. In addition, $f(u, \varphi)$ satisfies the following conditions:

$$
\lim _{s \rightarrow \infty} \frac{f\left(s, m_{2} s\right)}{s}=\infty, \quad \lim _{s \rightarrow 0} \frac{f\left(\sigma s, M_{2} s\right)}{s}=0,
$$

where

$$
m_{2}=\sigma^{\alpha} \min _{s \in[\theta, 1-\theta]} \int_{\theta}^{1-\theta} K(s, \tau) q(\tau) d \tau, \quad M_{2}=\int_{0}^{1} K(\tau, \tau) q(\tau) d \tau .
$$

Then system (1) has at least one positive solution.

Theorem 3.3 Assume (H1)-(H3), (H6) hold and $0<\alpha<1$. In addition,

(H8) $f \in C\left[R^{+} \times R^{+}, R^{+}\right]$, and $f(u, \varphi)$ is nondecreasing in $u$ and nonincreasing in $\varphi$ for $u, \varphi>0$. In addition, $f(u, \varphi)$ satisfies the following conditions:

$$
\lim _{s \rightarrow \infty} \frac{f\left(\sigma s, M_{3} s\right)}{s}=\infty, \quad \lim _{s \rightarrow 0} \frac{f\left(s, m_{3} s\right)}{s}=0,
$$

where

$$
m_{3}=\sigma^{\alpha} \min _{s \in[\theta, 1-\theta]} \int_{\theta}^{1-\theta} K(s, \tau) q(\tau) d \tau, \quad M_{3}=\int_{0}^{1} K(\tau, \tau) q(\tau) d \tau .
$$

Then system (1) has at least one positive solution.

Proof Since $\lim _{s \rightarrow 0} \frac{f\left(s, m_{3} s\right)}{s}=0$, there exists a $\eta>0$ with $\eta<1$ such that

$$
f\left(s, m_{3} s\right) \leq \epsilon s, \quad \text { for } 0<s<\eta,
$$

where $\epsilon$ satisfies

$$
\epsilon C \int_{0}^{1} G(s, s) h(s) d s \leq \frac{1}{2} .
$$


Taking $r \in(0, \eta)$ and setting $\Omega_{r}=\{u \in C[0,1]:\|u\|<r\}$, then, for any $u(t) \in \partial \Omega_{r} \cap P$, we have

$$
\begin{aligned}
T u(t)= & \int_{0}^{1} \int_{0}^{1} G(t, s) K(s, \tau) p(s) q(\tau) u(s) u^{\alpha}(\tau) d \tau d s \\
& +\int_{0}^{1} G(t, s) h(s) f\left(u, \int_{0}^{1} K(s, \tau) q(\tau) u^{\alpha}(\tau) d \tau\right) d s \\
= & C \int_{0}^{1} \int_{0}^{1} G(s, s) K(s, \tau) p(s) q(\tau)(\tau) d \tau d s \cdot r^{1+\alpha} \\
& +C \int_{0}^{1} G(s, s) h(s) f\left(r, \int_{0}^{1} K(s, \tau) q(\tau) u\left({ }^{\alpha} \tau\right) d \tau\right) d s \\
< & \frac{1}{2} r^{1+\alpha}+C \int_{0}^{1} G(s, s) h(s) f\left(r, \int_{\theta}^{1-\theta} K(s, \tau) q(\tau) u^{\alpha}(\tau) d \tau\right) d s \\
\leq & \frac{1}{2} r+C \int_{0}^{1} G(s, s) h(s) f\left(r, \int_{\theta}^{1-\theta} K(s, \tau) q(\tau) \sigma^{\alpha}\|u\|^{\alpha} d \tau\right) d s \\
\leq & \frac{1}{2} r+C \int_{0}^{1} G(s, s) h(s) f\left(r, \min _{s \in[\theta, 1-\theta]} \int_{\theta}^{1-\theta} K(s, \tau) q(\tau) \sigma^{\alpha}\|u\|^{\alpha} d \tau\right) d s \\
\leq & \frac{1}{2} r+C \int_{0}^{1} G(s, s) h(s) f\left(r, \min _{s \in[\theta, 1-\theta]} \int_{\theta}^{1-\theta} K(s, \tau) q(\tau) \sigma^{\alpha} d \tau \cdot r\right) d s \\
\leq & r=\|u\| .
\end{aligned}
$$

Consequently,

$$
\|T u\| \leq\|u\|, \quad \forall u(t) \in \partial \Omega_{r} \cap P .
$$

On the other hand, since $\lim _{s \rightarrow \infty} \frac{f\left(\sigma s, M_{3} s\right)}{s}=\infty$, there exists a $\bar{R}>0$ such that

$$
f\left(\sigma s, M_{3} s\right) \geq \rho s, \quad \forall s \geq \bar{R},
$$

where $\rho$ satisfies

$$
\rho \int_{\theta}^{1-\theta} G\left(\frac{1}{2}, s\right) h(s) d s>1 .
$$

Choosing $R>\left\{\frac{\bar{R}}{\sigma}, 2\right\}$ and setting $\Omega_{R}=\{u \in C[0,1]:\|u\|<R\}$, it is easy to see that

$$
\min _{t \in[\theta, 1-\theta]} u(t) \geq \sigma\|u\|=\sigma R>\bar{R}, \quad \forall u(t) \in \partial \Omega_{R} \cap P .
$$

Then, for all $u(t) \in \partial \Omega_{R} \cap P$, we have

$$
\begin{aligned}
\operatorname{Tu}\left(\frac{1}{2}\right)= & \int_{0}^{1} \int_{0}^{1} G\left(\frac{1}{2}, s\right) K(s, \tau) p(s) q(\tau) u(s) u^{\alpha}(\tau) d \tau d s \\
& +\int_{0}^{1} G\left(\frac{1}{2}, s\right) h(s) f\left(u, \int_{0}^{1} K(s, \tau) q(\tau) u^{\alpha}(\tau) d \tau\right) d s \\
\geq & \int_{0}^{1} G\left(\frac{1}{2}, s\right) h(s) f\left(u, \int_{0}^{1} K(s, \tau) q(\tau) u^{\alpha}(\tau) d \tau\right) d s
\end{aligned}
$$




$$
\begin{aligned}
& \geq \int_{\theta}^{1-\theta} G\left(\frac{1}{2}, s\right) h(s) f\left(u, \int_{0}^{1} K(s, \tau) q(\tau) u^{\alpha}(\tau) d \tau\right) d s \\
& \geq \int_{\theta}^{1-\theta} G\left(\frac{1}{2}, s\right) h(s) f\left(u, \int_{0}^{1} K(\tau, \tau) q(\tau) u^{\alpha}(\tau) d \tau\right) d s \\
& \geq \int_{\theta}^{1-\theta} G\left(\frac{1}{2}, s\right) h(s) f\left(\sigma\|u\|, M_{3}\|u\|^{\alpha}\right) d s \\
& =\int_{\theta}^{1-\theta} G\left(\frac{1}{2}, s\right) h(s) f\left(\sigma\|u\|, M_{3}\|u\|\right) d s \\
& \geq \int_{\theta}^{1-\theta} G\left(\frac{1}{2}, s\right) h(s) \rho\|u\| d s>\|u\| .
\end{aligned}
$$

Consequently,

$$
\|T u\| \geq\|u\|, \quad \forall u(t) \in \partial \Omega_{R} \cap P .
$$

Therefore, by Lemma 2.4, system (1) has at least one positive solution.

\section{Examples}

In this section, we only give some examples to illustrate the main theorems when $\lambda=0$ and $\theta=\frac{1}{4}$.

Example 4.1 Let us consider the following boundary value problem:

$$
\left\{\begin{array}{l}
-u^{\prime \prime}=t^{2} \varphi u+t\left(u^{2}+\varphi^{3}\right), \quad 0<t<1, \\
-\varphi^{\prime \prime}=u^{2}, \quad 0<t<1, \\
u(0)=u(1)=0 \\
\varphi(0)=\varphi(1)=0
\end{array}\right.
$$

where $\alpha=2, p(t)=t^{2}, q(t)=1, h(t)=t, f(u, \varphi)=u^{2}+\varphi^{3}$. Next via some simple computation, it is easy to see that $h(t):[0,1] \rightarrow[0,+\infty)$ is continuous, and

$$
\begin{aligned}
& \int_{0}^{1} G(s, s) h(s) d s=\int_{0}^{1} G(s, s) s d s=\int_{0}^{1} s(1-s) d s=\frac{1}{6}<\infty \\
& \int_{\frac{1}{4}}^{\frac{3}{4}} G\left(\frac{1}{2}, s\right) h(s) d s=\int_{\frac{1}{4}}^{\frac{3}{4}} G\left(\frac{1}{2}, s\right) s d s=\frac{3}{64}>0 .
\end{aligned}
$$

Then (H3) and (H5) hold. Since $p(t)=t^{2}$ and $q(t)=1$, we obtain

$$
\begin{aligned}
& C \int_{0}^{1} \int_{0}^{1} G(s, s) K(s, \tau) p(s) q(\tau) d \tau d s \leq \int_{0}^{1} \int_{0}^{1} G(s, s) K(\tau, \tau) p(s) q(\tau) d \tau d s=\frac{1}{120}<\frac{1}{2}, \\
& \min _{t \in\left[\frac{1}{4}, \frac{3}{4}\right]} \int_{\frac{1}{4}}^{\frac{3}{4}} K(t, s) q(s) d s=\frac{1}{16}>0 .
\end{aligned}
$$

So (H2) and (H4) hold. Finally, $f(u, \varphi)$ are nondecreasing in $u$ and $\varphi$ for $u, \varphi>0$, and

$$
\lim _{s \rightarrow \infty} \frac{f(s, m s)}{s}=\infty, \quad \lim _{s \rightarrow 0} \frac{f(s, M s)}{s}=0,
$$


where $m=\sigma^{\alpha-1} \min _{s \in\left[\frac{1}{4}, \frac{3}{4}\right]} \int_{\frac{1}{4}}^{\frac{3}{4}} K(s, \tau) q(\tau) d \tau=\frac{3}{256}, M=\int_{0}^{1} K(\tau, \tau) q(\tau) d \tau=\frac{1}{6}$. Thus (H6) holds. Therefore, from Theorem 3.1, the above problem has at least one positive solution.

Example 4.2 Let us consider the following boundary value problem:

$$
\left\{\begin{array}{l}
-u^{\prime \prime}=t^{2} \varphi u+t \frac{\varphi^{3}}{1+u} \\
-\varphi^{\prime \prime}=u^{2} \\
u(0)=u(1)=0 \\
\varphi(0)=\varphi(1)=0,
\end{array}\right.
$$

where $\alpha=2, p(t)=t^{2}, q(t)=1, f(u, \varphi)=\frac{\varphi^{3}}{1+u}$, and

$$
h(t)= \begin{cases}0, & 0 \leq t<\frac{1}{4} \\ -\left(t-\frac{1}{2}\right)^{2}+\frac{1}{16}, & \frac{1}{4} \leq t \leq \frac{3}{4} \\ 0, & \frac{3}{4}<t \leq 1\end{cases}
$$

Therefore, from Theorem 3.2, it is easy to see that the above problem has at least one positive solution.

Example 4.3 Let us consider the following boundary value problem:

$$
\left\{\begin{array}{l}
-u^{\prime \prime}=t^{2} \varphi u+t \frac{u^{2}}{1+\varphi} \\
-\varphi^{\prime \prime}=u^{\frac{1}{2}} \\
u(0)=u(1)=0 \\
\varphi(0)=\varphi(1)=0
\end{array}\right.
$$

where $\alpha=\frac{1}{2}, p(t)=t^{2}, q(t)=1, h(t)=t, f(u, \varphi)=\frac{u^{2}}{1+\varphi}$. Therefore, from Theorem 3.3, it is easy to see that the above problem has at least one positive solution.

Competing interests

The authors declare that they have no competing interests.

\section{Authors' contributions}

All authors contributed equally to the writing of this paper. All authors read and approved the final manuscript.

\section{Author details}

${ }^{1}$ College of Science, Hohai University, Nanjing, 210098, P.R. China. ${ }^{2}$ College of Mechanical Engineering, Guizhou Institute of Technology, Guiyang, 550003, P.R. China.

\section{Acknowledgements}

The first author is supported by NSF of China (No. 11501165), NSF of Jiangsu Province (No. BK20130825), the Fundamental Research Funds for the Central Universities. The second author is supported by Joint fund project of science and Technology Department of Guizhou Province, No. [2014]7366.

Received: 25 October 2015 Accepted: 30 December 2015 Published online: 13 January 2016

\section{References}

1. Gu, Y, Wang, M: Existence of positive stationary solutions and threshold results for reaction-diffusion system. J. Differ. Equ. 130, 277-291 (1996)

2. Wang, F, An, Y: Positive solutions for a second-order differential system. J. Math. Anal. Appl. 373, $370-375$ (2011)

3. Chen, $\mathrm{R}, \mathrm{Ma}$, R: Positive solutions of the second-order differential systems in reactor dynamics. Appl. Math. Comput. $219,3882-3892(2012)$ 
4. Chen, R, Ma, R: Global bifurcation of positive radial solutions for an elliptic system in reactor dynamics. Comput. Math. Appl. 65, 1119-1128 (2013)

5. Ruiz, D: The Schrödinger-Poisson equation under the effect of a nonlinear local term. J. Funct. Anal. 237(2), 655-674 (2006)

6. Jiang, Y, Zhou, H: Schrödinger-Poisson system with steep potential well. J. Differ. Equ. 249, 582-608 (2011)

7. Bonheure, D, Mercuri, C: Embedding theorems and existence results for nonlinear Schrödinger-Poisson systems with unbounded and vanishing potentials. J. Differ. Equ. 251, 1056-1085 (2011)

8. de Figueiredo, DG, Ruf, B: Elliptic systems with nonlinearities of arbitrary growth. Mediterr. J. Math. 1(4), 417-431 (2004)

9. Kcistaly, A: On a new class of elliptic systems with nonlinearities of arbitrary growth. J. Differ. Equ. 249, 1917-1928 (2010)

10. Guo, D, Lakshmikantham, V: Nonlinear Problems in Abstract Cones. Academic Press, New York (1988)

11. Li, Y: Positive solutions of fourth-order boundary value problems with two parameters. J. Math. Anal. Appl. 281, 477-484 (2003)

Submit your manuscript to a SpringerOpen ${ }^{\circ}$ journal and benefit from:

- Convenient online submission

Rigorous peer review

- Immediate publication on acceptance

Open access: articles freely available online

- High visibility within the field

- Retaining the copyright to your article 